\title{
Essential repairs
}

According to a study published in Science, OLIG1, a basic helix-loophelix transcription factor, is needed for the repair of demyelinating lesions that occur in multiple sclerosis. OLIG2 is already known to be required for the specification of oligodendrocytes and motor neurons during vertebrate embryogenesis. OLIG1 is coexpressed with OLIG2 in oligodendrocytes during development but, until now, its functions have remained a mystery.

Multiple sclerosis is caused by the death of mature oligodendrocytes, which leads to the loss of myelin sheaths. Fluctuating symptoms are a hallmark of this condition, and result from episodes of demyelination followed by the generation of new oligodendrocytes and remyelination. However, remyelination does not always occur, leading to the progressive deterioration that is characteristic of multiple sclerosis. Arnett and coworkers now show that the mechanisms that lead to remyelination in the adult brain parallel those that occur during the development of oligodendrocytes.

The authors first investigated the distribution of OLIG proteins during development. Both OLIG1 and OLIG2 were found in the nuclei of oligodendrocytes and oligodendrocyte

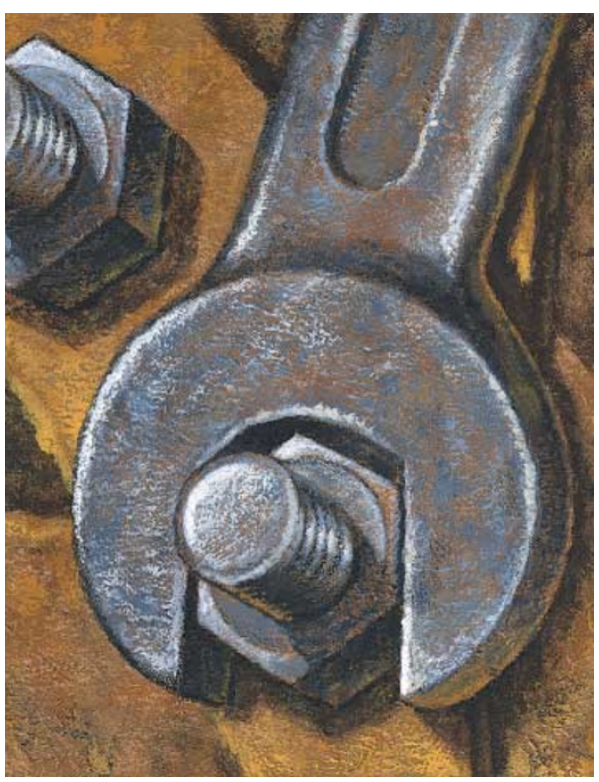

progenitor cells throughout the CNS of neonatal mice. However, whereas OLIG2 continued to be expressed in nuclei throughout development, OLIG1 was found mainly in the cytoplasm at 2 weeks after birth, and only in the cytoplasm of white matter in adult animals.

The next step was to investigate the distribution of OLIG proteins after demyelination. As in development, OLIG1 was found in the nuclei of cells within toxicity-induced demyelinating lesions in mice. Similarly, studies of post-mortem tissue from six patients with multiple sclerosis showed evidence for OLIG1 in the nuclei of oligodendrocytes in the white matter at the edges of active and chronic lesions, but in the cytoplasm of cells in normal white matter.

As remyelination is most likely to occur in active lesions, these data indicate that the relocation of OLIG1 to nuclei might be important for repair after white matter injury. This was confirmed by studies of mice that lacked OLIG1: a fully myelinated brain and spinal cord developed normally but there was little remyelination after toxicity-induced demyelination. Specifically, oligodendrocyte progenitor cells did not differentiate in these animals, underscoring a role for OLIG1 in differentiation of these cells and subsequent remyelination.

Therefore, intracellular localization of OLIG1 changes over the course of oligodendrocyte development, having a subtle effect during early development and a marked effect during remyelination. The parallels between the mechanisms involved in development and remyelination might help to identify a new therapeutic approach for multiple sclerosis.

Alison Rowan

(2) References and links ORIGINAL RESEARCH PAPER Arnett, H. A. et al. bHLH transcription factor Olig1 is required to repair demyelinated lesions in the CNS. Science 206, 2111-2115 (2004)

FURTHER READING

Franklin, R. J. M. Why does remyelination fail in multiple sclerosis? Nature Rev. Neurosci. 3 , 705-714 (2002)

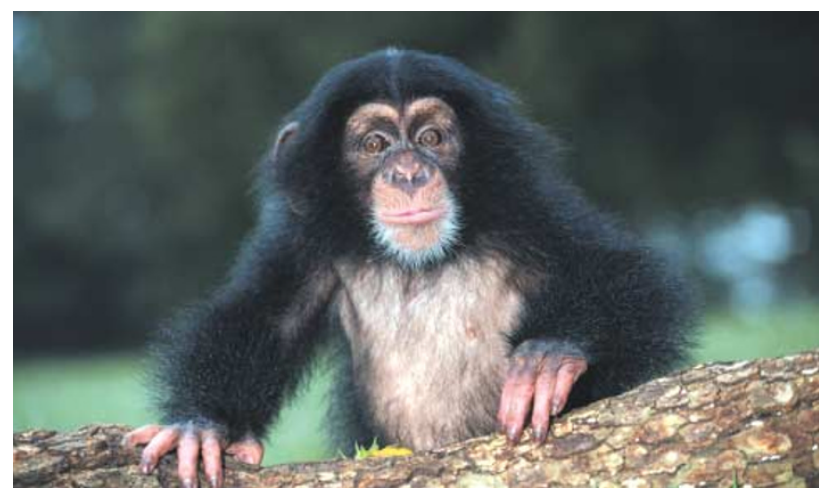

BRAIN EVOLUTION

\section{Fast-track to the human brain}

The remarkable increase in brain size and complexity during human evolution was accompanied by a marked acceleration in the evolution of genes that contribute to the development of the brain, according to a new study.

Primates have large and complex brains compared with other mammals, and this increased size and complexity reached its peak in humans. To investigate whether this rapid expansion was associated with accelerated evolution of specific genes, Dorus and colleagues compared the rates of evolution of 214 genes that have important roles in the nervous system in a primate lineage (between macaques and humans) and in another mammalian lineage (between rats and mice).

The average rate of evolution for these genes was much higher in the primate lineage than in the non-primate lineage. To check whether this was specific to genes that are involved in nervous system function, the authors carried out a similar comparison for 95 crucial 'housekeeping' genes, and found that primates and rodents had statistically identical rates of evolution for these genes. This supported the idea that the rapid evolution of nervous system genes in primates was due to positive selection.

To test this idea further, the authors looked specifically at a subset of nervous system genes - those that are important for the development of the nervous system. These genes showed an even greater difference in the rate of evolution between rodents and primates than did the larger set of nervous system genes. By contrast, genes that are important mainly for physiological functions in neurons or glia showed less acceleration of evolution. As developmental processes should be involved in evolutionary changes in brain size or complexity, the accelerated evolution of genes that are important for brain development supports the idea of positive selection associated with brain evolution.

Finally, the authors compared the lineage that led to humans with that leading to macaques. Human evolution involved much greater increases in brain size and complexity than did macaque evolution, and this was reflected in a much higher average rate of evolution for nervous system genes in the human lineage than the macaque one. So, not only have these genes evolved faster in primates than in other mammals, but in humans they have evolved fastest of all.

Rachel Jones

(2) References and links

ORIGINAL RESEARCH PAPER Dorus, S. et al. Accelerated evolution of nervous system genes in the origin of Homo sapiens. Cell 119, 1027-1040 (2004) WEB SITE Lahn laboratory http://hominid.uchicago.edu/ 